\title{
Spectroscopic transit search: a self-calibrating method for detecting planets around bright stars
}

\author{
Lennart van Sluijs ${ }^{1}$, Ernst de Mooij ${ }^{2}$, Matthew Kenworthy ${ }^{1}$, Maggie Celeste ${ }^{2}$, Matthew J. Hooton ${ }^{3}$, \\ Eric E. Mamajek ${ }^{4,5}$, Brigitta Sipőcz ${ }^{6}$, Ignas. A. G. Snellen ${ }^{1}$, Andrew R. Ridden-Harper ${ }^{7}$, and Paul A. Wilson ${ }^{8,9}$ \\ ${ }^{1}$ Leiden Observatory, PO Box 9513, 2300 RA Leiden, The Netherlands \\ e-mail: vansluijs@strw.leidenuniv.nl \\ ${ }^{2}$ School of Physical Sciences, and Centre for Astrophysics and Relativity, Dublin City University, Glasnevin, Dublin 9, Ireland \\ 3 Astrophysics Research Centre, School of Mathematics and Physics, Queen's University Belfast, Belfast BT7 1NN, UK \\ ${ }^{4}$ Jet Propulsion Laboratory, California Institute of Technology 4800 Oak Grove Dr., Pasadena, CA 91109, USA \\ ${ }^{5}$ Department of Physics and Astronomy, University of Rochester, 500 Wilson Blvd., Rochester, NY 14627, USA \\ ${ }^{6}$ Institute of Astronomy, University of Cambridge, Madingley Road, Cambridge, CB3 0HA, UK \\ ${ }^{7}$ Department of Astronomy, Cornell University, Ithaca, NY 14853, USA \\ ${ }^{8}$ Department of Physics, University of Warwick, Coventry CV4 7AL, UK \\ ${ }^{9}$ Centre for Exoplanets and Habitability, University of Warwick, Coventry CV4 7AL, UK
}

Received 15 January 2019 / Accepted 15 March 2019

\begin{abstract}
Aims. We aim to search for transiting exoplanets around the star $\beta$ Pictoris using high-resolution spectroscopy and Doppler imaging that removes the need for standard star observations. These data were obtained on the VLT with UVES during the course of an observing campaign throughout 2017 that monitored the Hill sphere transit of the exoplanet $\beta$ Pictoris b.

Methods. We utilized line profile tomography as a method for the discovery of transiting exoplanets. By measuring the exoplanet distortion of the stellar line profile, we removed the need for reference star measurements. We demonstrated the method with white noise simulations, and then looked at the case of $\beta$ Pictoris, which is a $\delta$ Scuti pulsator. We describe a method to remove the stellar pulsations and perform a search for any transiting exoplanets in the resultant data set. We injected fake planet transits with varying orbital periods and planet radii into the spectra and determined the recovery fraction.

Results. In the photon noise limited case we can recover planets down to a Neptune radius with an $\sim 80 \%$ success rate, using an $8 \mathrm{~m}$ telescope with a $R \sim 100000$ spectrograph and $20 \mathrm{~min}$ of observations per night. The pulsations of $\beta$ Pictoris limit our sensitivity to Jupiter-sized planets, but a pulsation removal algorithm improves this limit to Saturn-sized planets. We present two planet candidates, but argue that their signals are most likely caused by other phenomena.

Conclusions. We have demonstrated a method for searching for transiting exoplanets that (i) does not require ancillary calibration observations, (ii) can work on any star whose rotational broadening can be resolved with a high spectral dispersion spectrograph, and (iii) provides the lowest limits so far on the radii of transiting Jupiter-sized exoplanets around $\beta$ Pictoris with orbital periods from 15 days to 200 days with $>50 \%$ coverage.
\end{abstract}

Key words. methods: observational - techniques: spectroscopic - stars: individual: $\beta$ Pictoris - stars: variables: $\delta$ Scuti planets and satellites: detection

\section{Introduction}

A majority of the exoplanets discovered to date has been through the simultaneous photometric monitoring of several thousands of stars and a search for the decrement in stellar flux as a companion transits the stellar disk. Several ground-based photometric surveys, such as the Trans-Atlantic Exoplanet Survey (TESS; Alonso et al. 2004), XO (McCullough et al. 2005), Super Wide Angle Search for Planets (SuperWASP; Pollacco et al. 2006; Butters et al. 2010), Hungarian-made Automated Telescope Network (HATNet; Bakos et al. 2007), and NextGeneration Transit Survey (NGTS; Wheatley et al. 2018) and several space missions, such as Kepler (Borucki et al. 2010) and Convection, Rotation and planetary Transits (CoRoT) have been successful in detecting new exoplanets. Over two thousand transiting exoplanets have now been detected, and follow-up missions now include the TESS (Ricker et al. 2014) and PLAnetary Transits and Oscillations of stars (PLATO; Rauer et al. 2014) space missions. Transmission spectroscopy on these transiting exoplanets enables the characterization and detection of their atmospheres. The brighter the star, the higher is the signal to noise of the resultant exoplanet atmospheric spectrum (Seager \& Deming 2010). Finding the brightest star with a transiting exoplanet, therefore, is an important science goal that is being led from the ground by the WASP (Anderson et al. 2018), KELT (Lund et al. 2017), and MASCARA (Talens et al. 2017a, 2018) surveys, with MASCARA monitoring the brightest stars up to $V=4$ (Talens et al. 2017b). From space it is being led by TESS (Ricker et al. 2014), which also goes as bright as approximately $V=4$.

Ironically, despite the abundance of photons the brightest $V<4$ stars in the sky are not monitored by current transit surveys. This is largely because of the significant challenges in calibrating the photometry of bright stars in wide field surveys, detailed in Talens et al. (2017b). This is mostly due to the significantly different light paths from equally bright stars through 
the optics of a telescope and additionally, for ground-based telescopes, through the Earth's atmosphere. The limited field of view of larger telescopes means that it is very difficult to find a bright photometric standard with which to calibrate bright $V<4$ star transit observations.

Therefore, in this paper we present an alternative method for the detection of a transiting exoplanet that does not require a calibration star and thus can be utilized to survey the brightest $V<4$ stars in the sky, only requiring that they have sufficiently fast rotations. We look for the distortion of the rotationally broadened chromospheric stellar lines as a planet transits the stellar disk, also known as the Rossiter-McLaughlin (RM) effect. This technique is commonly used to determine the spin-orbit alignment of exoplanets and host stars during known transits (for an extensive overview, see Triaud 2017). Here we carry out a blind search for a transiting exoplanet using multi-epoch high spectral resolution observations of a bright star, calibrated using only the target star spectra. Many bright stars in the night sky are intermediate-mass main sequence stars. Their fast rotations (e.g., Gray 2005, and references therein) broaden the chromospheric lines, which limit their radial velocity sensitivity, and early type stars have typically far fewer absorption lines to provide a precise determination of their radial velocity.

In this paper we use $\beta$ Pictoris, a typical fast-rotating bright $(V<4)$ star, to investigate the feasibility of this method. The nearby (van Leeuwen 2007) bright young ( 23 Myr; Mamajek \& Bell 2014) A6V star $\beta$ Pictoris has both a debris disk and at least one giant planet, $\beta$ Pictoris b (Lagrange et al. 2009, 2019) in orbit around it. Both the disk and the planet are seen nearly edge-on with a very high inclination of $>89^{\circ}$ (Wang et al. 2016). In 2017 the Hill sphere of the planet moved in front of the star, taking approximately 200 days to move across in a chord that brought the line of sight of the star to within $20 \%$ of the Hill sphere radius. A comprehensive campaign of photometric and spectroscopic observations was taken over this period, searching for circumplanetary material (Kenworthy 2017). Due to the abundance of high-resolution spectra available as part of this campaign (PI: E. de Mooij), $\beta$ Pictoris is an excellent target to study the feasibility of our method.

Firstly, in Sect. 2 we outline the method by studying its potential by simulating observations of a transiting companion around a fast rotating $V \sim 4$ bright star. Secondly, in Sect. 3 we apply our method to real data of $\beta$ Pictoris as a case study. The results are discussed in Sect. 4 followed by our conclusions and future prospects in Sect. 5.

\section{Principle of the method}

In this section we demonstrate the application of the RM effect to the discovery of new planets. For this we first explain how the RM effect is modeled in the next subsection and then highlight its ability to recover planetary signals using a set of white noise simulations of a bright $(V \sim 4)$ star with a high-resolution spectrograph on an $8 \mathrm{~m}$ telescope.

\section{1. $R M$ model}

Our RM model is based on the model used by de Mooij et al. (2017), and uses a grid-based method to calculate the line profile of a rotating star. For the model we assume solid body rotation, quadratic limb darkening, and no gravity darkening. The intrinsic line profile, $F_{i j}(v)$, at a pixel location $(i, j)$, is modeled as a Gaussian with a line-depth $A$, a width given by the full width at half maximum, FWHM. For each pixel, the line profile is centered on a radial velocity $v_{\text {rot }, i j}$, due to the stellar rotation at that position. The planet is modeled as a black disk at position $\left(i_{\mathrm{p}}, 0\right)$, assuming an orbit parallel to the $x$-axis and a projected spin-orbit misalignment $\lambda=0^{\circ}$ with impact parameter $b=0$. For all our simulations, we use a grid of 1025 by 1025 spatial pixels for the calculations of the spectrum with a stellar radius of 510 pixels. To reduce the impact of aliasing effects, especially for smaller planets and at ingress and egress, both the stellar intensity map and the planet map are initially calculated on a grid that is over-sampled by a factor of ten in both directions, and rebinned to 1025 by 1025 pixels before calculating the final spectrum. This spectrum is calculated on a velocity grid of $3 \mathrm{~km} \mathrm{~s}^{-1}$ steps.

\subsection{White noise simulation}

We demonstrate the method by considering photon-shot-noiselimited simulated observations of a typical bright star that shows rotationally broadened spectral lines resolved with a highresolution spectrograph. We take the parameters for an $\mathrm{A6V}$ star of magnitude $V=4$ observed with an $8 \mathrm{~m}$ telescope using a high-resolution spectrograph and assume a signal-to-noise $(\mathrm{S} / \mathrm{N})$ of $1200 \mathrm{pixel}^{-1}$. Simulated observations are created by adding white noise at this spectral $\mathrm{S} / \mathrm{N}$ to the normalized line profiles. We assume 21 spectra are taken over a $30 \mathrm{~min}$ period per individual night, and that there are a total of 152 nights of observations. Lastly, in line with the values for a typical A6V star from de Mooij et al. (2017), we assume an intrinsic line width of $20 \mathrm{~km} \mathrm{~s}^{-1} F W H M$, projected equatorial stellar rotation $v_{\mathrm{eq}}=130 \mathrm{~km} \mathrm{~s}^{-1}$ (sometimes also referred to as $v \sin i$ ), $V$-band limb darkening coefficients for an effective temperature of $8000 \mathrm{~K}$ and $\log g=4.0$ (Claret 2000), and an intrinsic line depth $A=0.8$. We create simulated residual spectra (after median line profile subtraction) at our spectral $\mathrm{S} / \mathrm{N}$ for the full observation window, and then we apply the following steps to calculate the exoplanet $\mathrm{S} / \mathrm{N}$ at different stellar positions and exoplanet radii:

- an exoplanet with a given radius is injected into one night of spectra with impact parameter $b=0$, at a given radial velocity offset;

- the exoplanet signal is calculated by summing up all the flux in $24 \mathrm{~km} \mathrm{~s}^{-1}$ (eight-pixel-wide) bins;

- the noise is estimated as the standard deviation of the signals over all the other nights, where no signal was injected. We assume only one planetary transit occurs in the data.

This routine is repeated for all nights and with varying of the injected planet radius and radial velocity offsets. A planet is said to be recovered if the $S / N>3.0$ for the injected planet's location. The recovery fraction is the number of recovered planets normalized by the total number of nights. The result is shown in Fig. 1. We compare the transiting exoplanet radius $R$ to the radius of Jupiter $R_{\text {Jup }}$, Saturn $R_{\text {Sat }}$, and Neptune $R_{\text {Nep }}$. Companions with radii $R>R_{\text {Nep }}$ are fully recovered and radii $R=R_{\text {Nep }}$ are recovered $\sim 80 \%$ of the time. For the latter, the recovered fraction is less for larger radial speeds. This is due to limb darkening, which causes a weaker line profile distortion towards the stellar edges.

\subsection{Period completeness and coverage}

Our coverage, $\operatorname{Cov}(R, P)$, is the product of the sensitivity, $\operatorname{Sen}(R)$, which is the probability of detecting an exoplanet of size $R$, and the period completeness $\operatorname{Com}(P)$, which is the probability of detecting a transit given our observation window. The sensitivity is averaged over all radial velocity offsets in Fig. 1. The period 


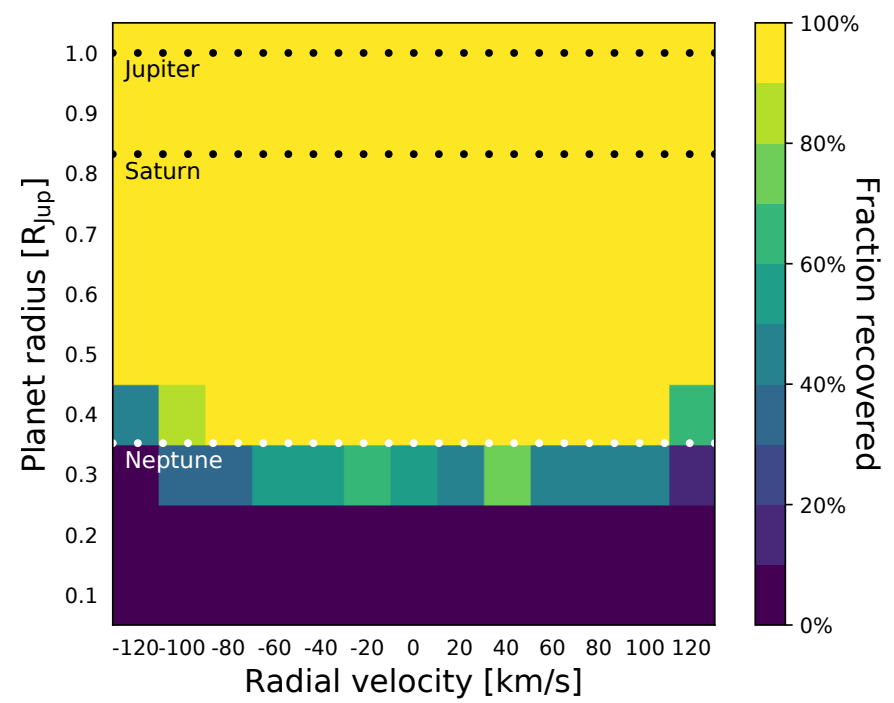

Fig. 1. Recovery fraction of transit injections in 152 nights for our white noise simulation. Exoplanet signals with a $S / N>3.0$ above other nights are considered recovered. We assume an impact parameter $b=0$ and a star observed at $S / N=1200 \mathrm{pix}^{-1}$. The radii of Jupiter, Saturn, and Neptune are indicated by the black and white dashed lines. As $v_{\mathrm{eq}}=130 \mathrm{~km} \mathrm{~s}^{-1}$, radial velocities outside of our range are (close-to) zero. The effects of limb darkening are seen in the bottom rows as lower recovery fractions for higher radial speeds.

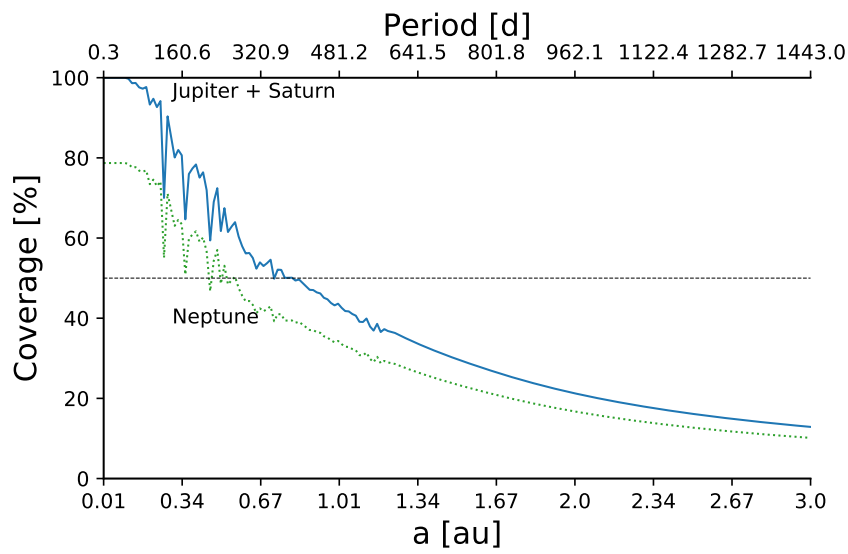

Fig. 2. Coverage of our observation window for Jupiter-, Saturn-, and Neptune-sized object for our white noise simulation assuming an impact parameter $b=0$. The period completeness equals the Jupiter and Saturn coverage as their sensitivity is $100 \%$. For longer periods, the period completeness decreases with decreasing slope. The horizontal dashed line indicates $50 \%$ coverage.

completeness depends only on the observation window and is calculated in the following way:

- the transit duration is calculated;

- the observation window function is convolved with the transit duration;

- this convolved window function is folded to the exoplanet period;

- the coverage is calculated by taking the ratio of non-zero values over zero values of the period folded convolved window function.

The coverage results in this photon-shot-noise-limited case are shown in Fig. 2. For radii $R \geq R_{\text {Sat }}$, the sensitivity is $100 \%$, thus the coverage coincides with the period completeness. Periods $<13 \mathrm{~d}(\sim 0.13 \mathrm{au})$ are fully complete. For longer periods the completeness decreases with decreasing slope. This is due to longer transit durations for longer periods. Periods $\sim 1 \mathrm{yr}(\sim 0.8 \mathrm{au})$ have a 50\% completeness and periods $\sim 1400 \mathrm{~d}(\sim 3 \mathrm{au})$ have a $\sim 10 \%$ completeness. Small coverage fluctuations are seen for periods $<1 \mathrm{au}$. This is due to the non-uniformity of our window function, which causes an overlap of the observation windows when folded for certain periods. Nonetheless, this effect is negligible, as the resolved gaps have a FWHM of $\sim 0.1 \mathrm{~d}$. In contrast to photometric light curve transits, we do not need to follow the whole transit from ingress to egress. Instead, the spectral line profile distortion determines the diameter of the transiting object.

\section{Application to real data: search for transiting planets orbiting $\beta$ Pictoris}

Section 2 describes an idealized scenario. In this section we apply our method to analyze high-spectral-resolution observations of $\beta$ Pictoris obtained in 2017-2018.

\subsection{Observations}

We obtained observations on 160 epochs between Apr 1, 2017 and Apr 17, 2018 with the Ultraviolet and Visual Echelle Spectrograph (UVES) at the Very Large Telescope (VLT) in Chile (Dekker et al. 2000). During each observation the data were obtained simultaneously with the red and the blue arm using the \#2 dichroic and the CD4 and CD2 cross dispersers in the two arms, respectively. For the wavelength mode the $437+760$ mode was selected, resulting in a wavelength coverage of 3760-4980 $\AA$ and 5700-9450 $\AA$ in the blue and red arms, respectively. Due to the large amount of telluric lines in the red arm, we focus only on data in the blue arm in this paper. We used a $0.3^{\prime \prime}$ slit to obtain the highest possible resolution ( 20000 before an instrument intervention by the observatory in Oct 2017, which resulted in an increase in resolving power to 100000). The exposure time for the blue arm was $15 \mathrm{~s}$. For the first ten epochs, we used the $225 \mathrm{kHz}$ readout mode, and obtained 15 exposures per visit; for the remainder we used the $625 \mathrm{kHz}$ mode and obtained 21 exposures per visit.

The data were initially reduced using the UVES pipeline version 5.7.0 via the ESO Reflex (Freudling et al. 2013), and we used the spectra before merging of the individual orders for the remainder of our analysis. After the initial reduction, the spectra were interpolated onto a common wavelength grid and corrected for blaze variations between spectra and nights. The blaze correction was done on a frame-by-frame and order-by-order basis by fitting a low-order polynomial to the ratio of the frame and echelle order being considered and the same echelle order in the reference frame. For the reference frame, the average spectrum for the sixth visit was used. After blaze correction, we selected 16 lines across the different orders that visually appeared to be unblended, and cut out a region of $\sim 215 \mathrm{~km} \mathrm{~s}^{-1}$ around the apparent line center. To improve the $\mathrm{S} / \mathrm{N}$ and to allow the individual lines to be combined directly, we binned the data onto a velocity grid with a pixel size of $3 \mathrm{~km} \mathrm{~s}^{-1}$. After visual inspection of all nights, we flagged eight nights due to bad data quality, making in total 152 nights available for further analysis. The flagged nights are (UT): 2017 Apr 8, Sep 11, Oct 5, Oct 8, Oct 9, Oct 10, Oct 13, and 2018 Feb 3.

\subsection{Stellar pulsations}

In contrast to our white noise simulations presented in Sect. 2.2, $\beta$ Pictoris is a $\delta$ Scuti non-radial pulsator (Koen et al. 2003), which will have a direct impact on our sensitivity. For a given set of spectra on a single night, the median stellar profile of all of 


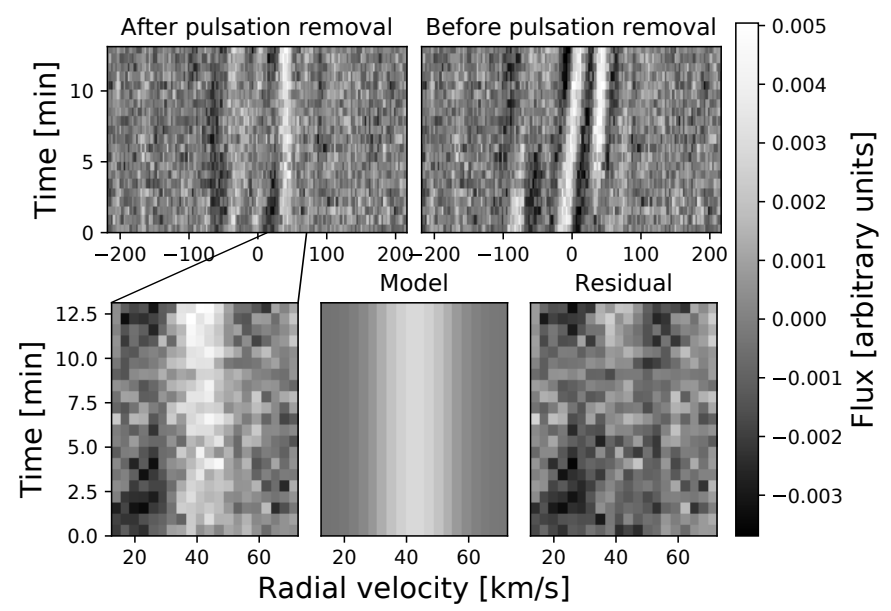

(a)

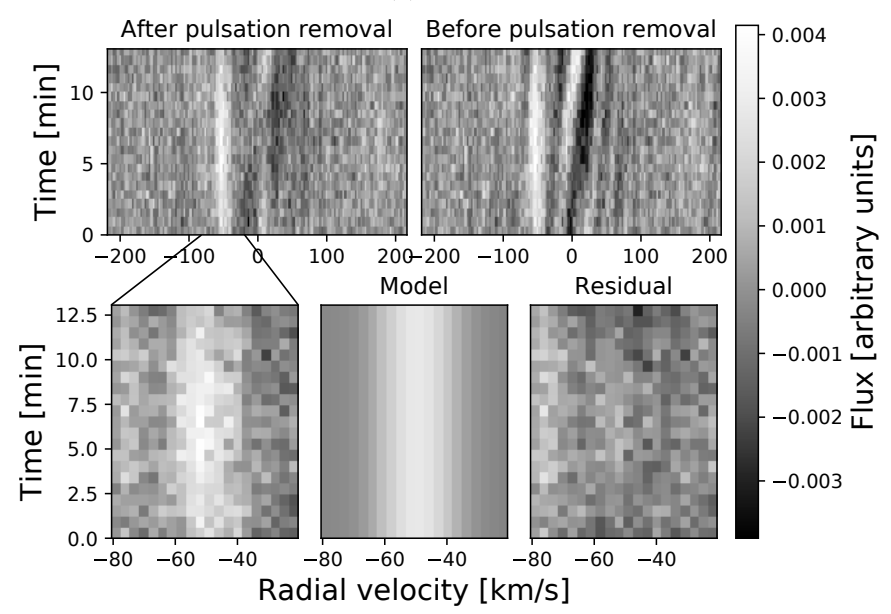

(b)

Fig. 3. Spectral time series of two nights after median line profile subtraction and combining 16 lines. Panel $a$ : exoplanet candidate for the night of UT 2017 Sep 8 (JD=2458004). Panel $b$ : exoplanet candidate for the night of UT 2017 Dec 11 (JD = 2458098). The $\delta$ Scuti pulsations can be seen in the top right boxes and differ in amplitude, shape, and slope. Two exoplanet candidate signals are boosted after pulsation removal. Zooms of the signals, RM models, and residuals are shown in the bottom three boxes.

the other nights are subtracted from the current night. The stellar pulsations appear as a quasi-sinusoidal signal in velocity space that change as a function of time. The peak of a given pulsation in velocity space is assumed to vary linearly with observing time. If the peak amplitudes do not change, they appear as vertical black and white stripes in the residual spectral line time sequence, as seen in the upper right boxes of Figs. $3 a$ and $b$.

The pulsation amplitudes are similar to those expected for approximately Jupiter-sized exoplanet signals, thus pulsation removal is required to detect smaller radii companions. This pulsation removal has been done successfully for broadband time series photometric observations (Zwintz 2018; Mol Lous et al. 2018) and for spectral observations with a companion on a retrograde short-period (1-2 d) orbit (Johnson et al. 2015; Temple et al. 2017). However, this has not been done before for spectral observations of companions on prograde orbits or on retrograde orbits with $>2 \mathrm{~d}$ periods. We have developed a method to perform the pulsation removal ${ }^{1}$, which we present in

\footnotetext{
1 Our pipeline is publicly available at https://github.com/ lennartvansluijs/Spectroscopic-Transit-Search
}

this subsection. We provide the results on the detection limits and coverage, including any residual pulsations, for $\beta$ Pic in Sect. 3.3. The main difficulty is the degeneracy between the stellar pulsations and exoplanet signals in the spectral time series. A stellar pulsation model using many free parameter fits can overfit an exoplanet signal, whereas using fewer free parameters does not fit the pulsations well when our pulsation model breaks down. We tried the following approaches: (i) principle component analysis (PCA) on all spectra and removal of the most dominant eigenvectors within the spectra, (ii) sinusoidal-fitting per nigh,t (iii) shearing the spectra and applying PCA, (iv) shearing every night and subtracting the mean along the time- axis, and (v) same as (iv) but with an extra correction for the contribution of an exoplanet signal. The last method resulted in the best $\mathrm{S} / \mathrm{N}$ improvement, and we describe it below.

The radial velocity change during one observation $(\sim 10$ 20 min observing time) $\Delta v_{\text {obs }}$ due to stellar pulsations is $\sim 30$ $50 \mathrm{~km} \mathrm{~s}^{-1}$. The change in radial velocity due to the planet blocking out different velocity strips of the stellar surface $\Delta v_{\text {obs }}$ of an edge-on and aligned planet for an observation duration $t_{\mathrm{obs}}$ and transit duration $t_{\text {transit }}$ is

$\Delta v_{\text {obs }}=2 v_{\text {eq }}\left(\frac{t_{\text {obs }}}{t_{\text {transit }}}\right) \quad\left(t_{\text {obs }} \leq t_{\text {transit }}\right)$.

Therefore a hypothetical planet on a 0.1 au orbit around $\beta$ Pictoris would show a shift of $\Delta v_{\text {obs }} \approx 9 \mathrm{~km} \mathrm{~s}^{-1}$ in a typical 30 min observation. This number will be even smaller for longer orbital periods, so an exoplanet radial velocity signal is approximated as being constant during the $30 \mathrm{~min}$ of spectra. The planet signal appears as a dark vertical stripe in the time series of spectra of $30 \mathrm{~min}$. However, transiting objects on prograde short period orbits will have slopes aligned with the stellar pulsation signals, making it more difficult to detect them. On the contrary, such objects on retrograde orbits are easier to detect as they skew from the stellar pulsation signals (as for the Doppler shadow in the HD 15082 (WASP 33) system; Collier Cameron et al. 2010). The difference in $\Delta v_{\text {obs }}$ between an exoplanet and pulsation signal can be exploited by "shearing" the spectra as shown in Fig. 4: shifting each spectrum $i$ observed at epoch $t_{i}$ in radial velocity space $\Delta v_{i}$ proportional to their time difference $\Delta t_{i}$ with the midtime epoch of the nightly set of spectra $t_{0}$. We define the shearing constant $S$ in $\Delta v_{i}=S t$ where $t=t_{i}-t_{0}$. This aligns most of the stellar pulsations and shears them into vertical lines, aiding their estimation and subsequent modeling and removal. For the stellar pulsation removal, the following steps are applied as shown in Fig. 4:

- positive velocity shearing $+S$ is applied for differing values of the shearing constant (third column in Fig. 4);

- the exoplanet signal (red) is estimated for all different shears by applying an equal and opposite shearing (first column);

- the exoplanet signal estimates are subtracted (third column minus first column);

- the pulsations are estimated by the residual of the positive shearing for which the pulsations aligned the best (blue line in third column);

- the pulsation estimate is subtracted from the sheared data;

- the shearing is reversed $(-S)$ and the spectra summed in the time direction.

The final result for our mock data example is shown in the rightmost column of Fig. 4: a clear $\mathrm{S} / \mathrm{N}$ improvement with respect to the central column. 

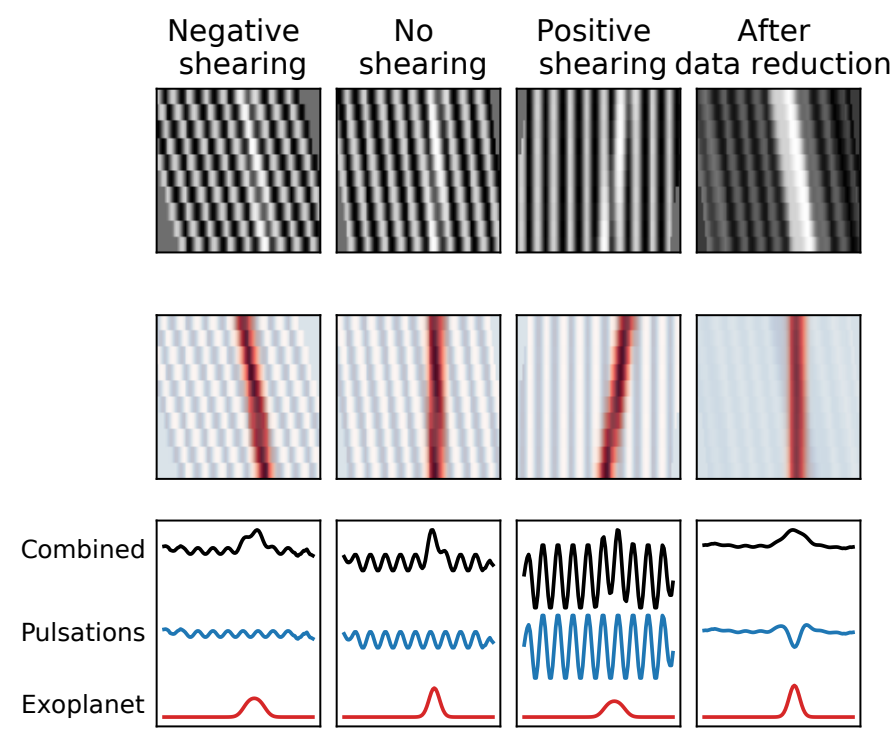

Fig. 4. Illustration of our pulsation removal algorithm. Black is the observed combined signal, blue the pulsation component (modeled by an inclined sinusoidal signal), and red the exoplanet component (modeled by a Gaussian signal). Positive shearing of the spectra aligns the pulsations. Due to symmetry, an equal negative shearing estimates the exoplanet signal for the positive shearing. Subtraction of the estimated exoplanet and pulsation signal then reverse shearing boosts our exoplanet signal and suppresses pulsations.

\subsection{Sensitivity and coverage}

Residual spectra for all observations of $\beta$ Pictoris are created the following way:

- the median line profile of all nights for all lines is calculated and used as a reference line profile;

- all line profiles are normalized with respect to the reference line profile;

- the reference line profiles are subtracted.

The same planet injection routine is applied, combined with our pulsation removal routine. The results are shown in Fig. 5. Companions with $R=R_{\mathrm{Jup}}$ are recovered for $\sim 74 \%$ of all nights. The recovered fraction of $R=R_{\text {Sat }}$ companions is $\sim 36 \%$. As the stellar and observational parameters described in Sect. 2.2 match with $\beta$ Pictoris, we can directly compare Figs. 1 and 5. This shows pulsations limit our sensitivity to Saturn- to Neptunesized objects. Using these sensitivity limits we are able to calculate coverage limits as described in Sect. 2.3, with the results shown in Fig. 6. Neptune-sized objects in the photon noise limit and Jupiter-sized objects in the pulsation limit have almost equal coverage, which shows the direct impact of the stellar pulsations on our sensitivity.

\subsection{Comparison with previous surveys}

One previous exoplanet transit search around $\beta$ Pictoris was conducted using data from the BRIght Target Explorer (BRITE)Constellation nanosatellite BRITE-Heweliusz (Mol Lous et al. 2018). Their coverage for periods $\leq 15 \mathrm{~d}$ is similar to ours for $R=R_{\mathrm{Jup}}$ and slightly better for $R=R_{\mathrm{Sat}}$. For periods $\geq 15 \mathrm{~d}$ our coverage is better. This is partially due to the difference in completeness: 78 nights for Mol Lous et al. (2018) and 152 nights for this work. Nonetheless, for a conventional transit survey detection, coverage of the full transit is required. Our proposed method does not require full transit coverage, as coverage of only part of the transit will already reveal a spectral line profile

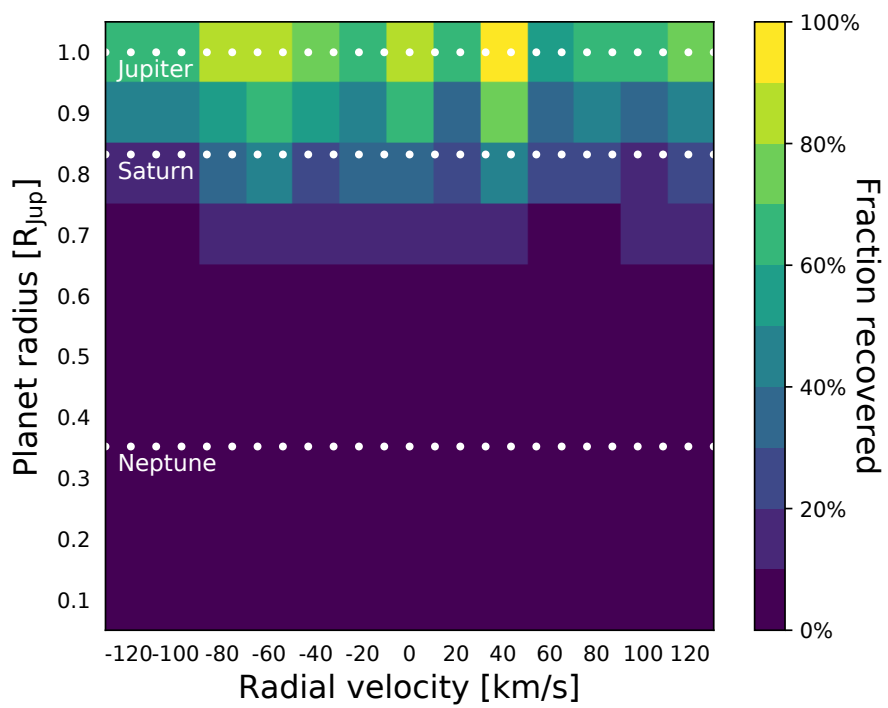

Fig. 5. Recovery fraction of 152 transit injections into the $\beta$ Pictoris dataset. Exoplanet signals with a $S / N>3.0$ above other nights are considered recovered. We assume an impact parameter $b=0$. The radii of Jupiter, Saturn and Neptune are indicated by the white dashed lines. As $v_{\mathrm{eq}}=130 \mathrm{~km} \mathrm{~s}^{-1}$, radial velocities outside of our range are (close-to) zero. Companions with radii $R=R_{\text {Jup }}$ are almost fully recovered and a large fraction with radii $R=R_{\text {Sat }}$ are recovered. The effects of stellar pulsation are seen by the fluctuations of the recovery fraction as a function of radial velocity.

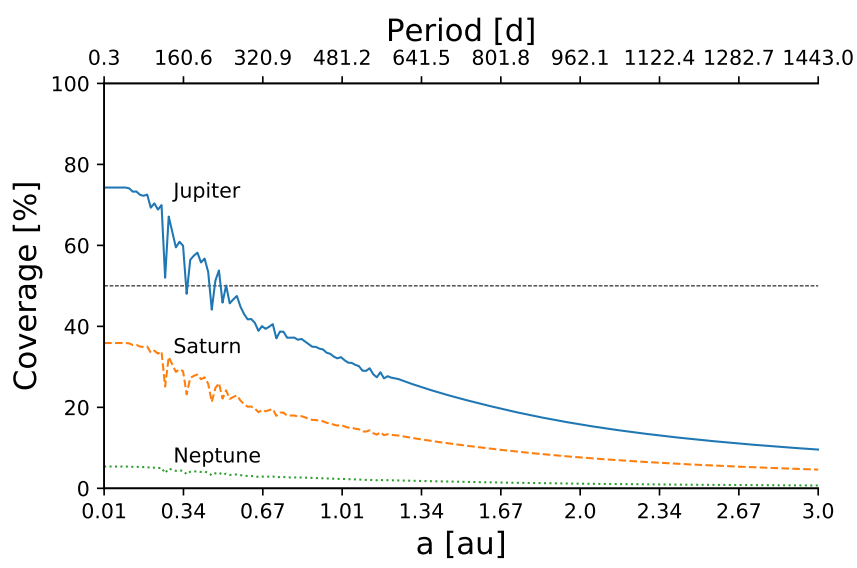

Fig. 6. Coverage for our observation window for Jupiter-, Saturn- and Neptune-sized objects in the stellar pulsation-limited case for an impact parameter $b=0$. For longer periods, the coverage drops with decreasing slope. The horizontal dashed line indicates $50 \%$ coverage.

distortion. This is an advantage of our method over conventional transit surveys. Additionally, exoplanet radial velocity studies have been done by Lagrange et al. (2013, 2018). Following Mol Lous et al. (2018), the mass upper limits at different orbital periods can be converted into radii using FORECASTER ${ }^{2}$ (Chen \& Kipping 2017). Compared to Lagrange et al. (2013), the sensitivity increases significantly for $R<R_{\text {Jup }}$ objects at smaller orbital periods. One major result of Lagrange et al. (2018) is the exclusion of companions more massive than $3 M_{\text {Jup }}$ closer than $1 \mathrm{au}$ and further than $10 \mathrm{au}$, with a $90 \%$ probability. Even in the fully recovered case, we have a coverage of $>45 \%$ at 1 au (see Fig. 2), less than $90 \%$. For a very massive transiting companion

2 Available at https://github.com/chenjj2/forecaster 


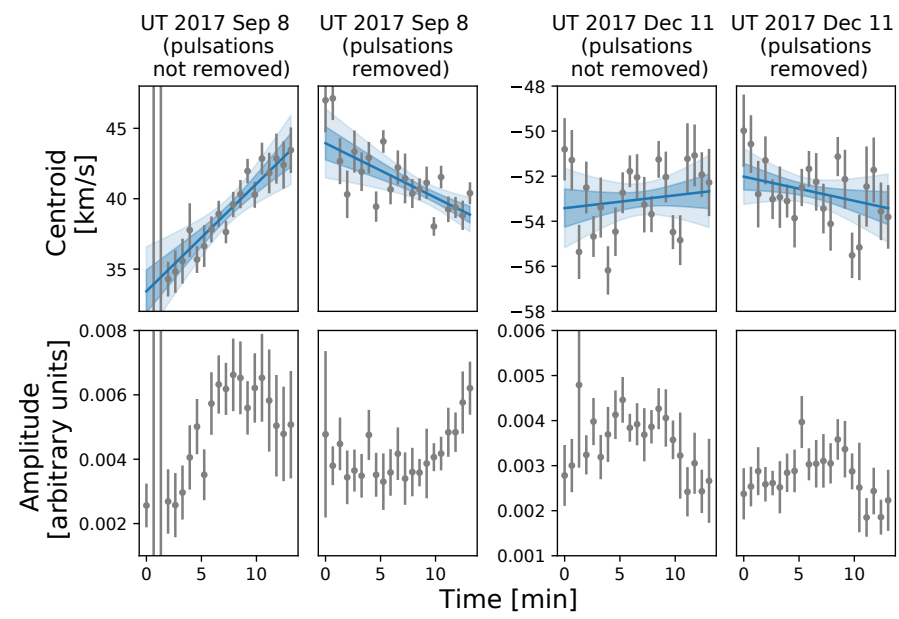

Fig. 7. Gaussian with variable background best-fits to the spectral time series of the two candidates in Fig. 3. This has been done both before and after stellar pulsation removal. The best linear fit of the centroid time series and corresponding $1 \sigma$ - and $2 \sigma$-confidence intervals are shown in blue and light blue. The bottom row shows the best-fit amplitude time series.

$<1$ au and at very long orbital periods ( $\gtrsim 200 \mathrm{~d})$, the radial velocity search has greater sensitivity. For the latter, as geometrical transit probabilities are very small in this regime, it is unlikely any future transit survey will be competitive in this regime.

\subsection{Candidates}

A similar analysis search in our data results in 12 nights containing a signal with a $S / N>3.0$. Visual inspection shows most of these are due to strong outlier pixels, strong stellar pulsations, or bad data quality. Two candidates remain (see Fig. 3); for both, a $R=R_{\text {Jup }}$ at an edge-on orbit object fits the signal well. Gaussian profiles with variable background are fitted using Levenberg-Marquardt minimization to each spectral time series, both before and after stellar pulsation removal, to obtain an amplitude and centroid time series. A linear fit to the latter constrains the Doppler shadow's slope. The results are shown in Fig. 7. After pulsation removal, we find $\Delta v_{\text {rad }}=-5.1 \pm 1.6 \mathrm{~km} \mathrm{~s}^{-1}$ (Sep 8, 2017) and $\Delta v_{\text {rad }}=-1.4 \pm 1.2 \mathrm{~km} \mathrm{~s}^{-1}$ (Dec 11, 2017), both suggesting retrograde orbits. Orbital period limits are calculated using Eq. (1). We find $P=0.2_{-0.1}^{+0.3} \mathrm{yr}$ (Sep 8, 2017) and $P=6.0_{-4.8}^{+\inf }$ yr (Dec 11, 2017). For the candidate of Sep 8, 2017 a detection is plausible based on the period lower limit, for which we have $\gtrsim 75 \%$ coverage. However, the amplitude time series shows a strong increase in amplitude over the observational duration, which is expected for the stellar pulsations with short periods, but not for a transiting companion. This is supported by the radial velocity change before pulsation removal of $\Delta v_{\text {rad }}=10 \pm 2.5 \mathrm{~km} \mathrm{~s}^{-1}$, much closer to typical stellar pulsation values. For the candidate of Dec 11, 2017 it is plausible with the period lower limit, for which we have $\gtrsim 35 \%$ coverage. The slope in the velocity during the observations is consistent both before and after pulsation removal with an exoplanet signal, however, the amplitude time series shows a significant variation, suggesting that the signal is not planetary in nature. Consequently, we conclude both signals are unlikely due to a transiting exoplanet. The retrieval of these two signals demonstrates the capability of our algorithm to retrieve exoplanet-like signals and the subsequent analysis shows it is possible to identify false positives from their slope and time variation. We find these results very
Table 1. Stellar parameters used for the white noise simulations of different stellar spectral types.

\begin{tabular}{lccr}
\hline \hline Spectral type & Radius $\left[R_{\odot}\right]$ & $v_{\text {eq }}\left(\mathrm{km} \mathrm{s}^{-1}\right)$ & Teff $(\mathrm{K})$ \\
\hline B0 & 7.53 & 350 & 31500 \\
B5 & 3.40 & 330 & 15700 \\
A0 & 2.09 & 310 & 9700 \\
A5 & 1.94 & 290 & 8080 \\
F0 & 1.79 & 170 & 7220 \\
F5 & 1.46 & 40 & 6510 \\
\hline
\end{tabular}

encouraging, but this also demonstrates the detection of at least three transits will be required to confirm the planetary nature of the signals in the future.

\section{Discussion}

In the previous sections we have shown the principle of the method for a typical bright star, $\beta$ Pictoris. In this section we discuss the possibility of applying our method to other bright stars in the sky. Therefore, we extend our analysis to a broader range of spectral types and instrumental parameters. According to the Set of Identifications, Measurements and Bibliography for Astronomical Data (SIMBAD) database (Wenger et al. 2000), there are 512 stars with $V<4$ observed by HIPPARCOS (Perryman et al. 1997). Among these, a majority of 272 stars are BAF spectral types. These are expected to rotate quickly enough to resolve the planet Doppler shadow, but have stellar radii small enough to detect exoplanets. For these spectral types we simulated line profiles of planets transiting in front of the stellar center. These profiles were calculated on an oversampled velocity grid of $1 \mathrm{~km} \mathrm{~s}^{-1}$ steps. We adopted the stellar radii and temperatures by Pecaut \& Mamajek $(2013)^{3}$ as shown in Table 1. A future survey would likely aim to search for companions around the fastest rotating stars first, as (i) they are more likely to have an edge-on inclination thus a transiting companion and (ii) it is easier to resolve the spectral line profiles. Therefore, we follow the upper bounds on the $v_{\text {eq }}$ values for each spectral type estimated from Fig. 18.21 from Gray (2005) (see Table 1). The intrinsic line width $v_{\text {int }}$ due to thermal broadening is described by the Maxwell-Boltzmann distribution $v_{\text {int }} \propto \sqrt{T}$. Using the temperatures in Table 1 a simple scaling relation is adopted to estimate $v_{\text {int }}$ for all spectral types where we benchmark at $v_{\text {int }}=20 \mathrm{~km} \mathrm{~s}^{-1}$ and $T=8000 \mathrm{~K}$. These over-sampled line profiles are convolved and binned to an instrumental spectral resolution $\lambda / \Delta \lambda$. The planet signal is measured as the sum of all points where the signal is above zero in the out-of-transit subtracted line profile. Assuming an instrumental spectral S/N per resolution element, we calculated the $\mathrm{S} / \mathrm{N}$ of the exoplanet signal. The results are shown in Fig. 8. The contours show the $S / N>3.0$ limits for the different spectral types. For larger stellar radii, the amplitude of the exoplanet signal is smaller. For larger rotational velocities, a lower resolution is required to resolve the exoplanet signal. This effect is most prominent for the F5-spectral type, which has the lowest $v_{\text {eq }}$ value. For stars with spectral resolutions and $\mathrm{S} / \mathrm{Ns}$ to the right of the line, it is feasible to detect a companion of the specified size. Consistent with our previous result, the $\beta$ Pictoris (an A6 star) observations are on the right side of the A5 star $R=R_{\text {Nep }}$ contour, as we are sensitive to $R=R_{\text {Nep }}$ companions in the photon-shot-noise-limited case.

\footnotetext{
3 http://www.pas.rochester.edu/ emamajek/EEM_dwarf_
} UBVIJHK_colors_Teff.txt 


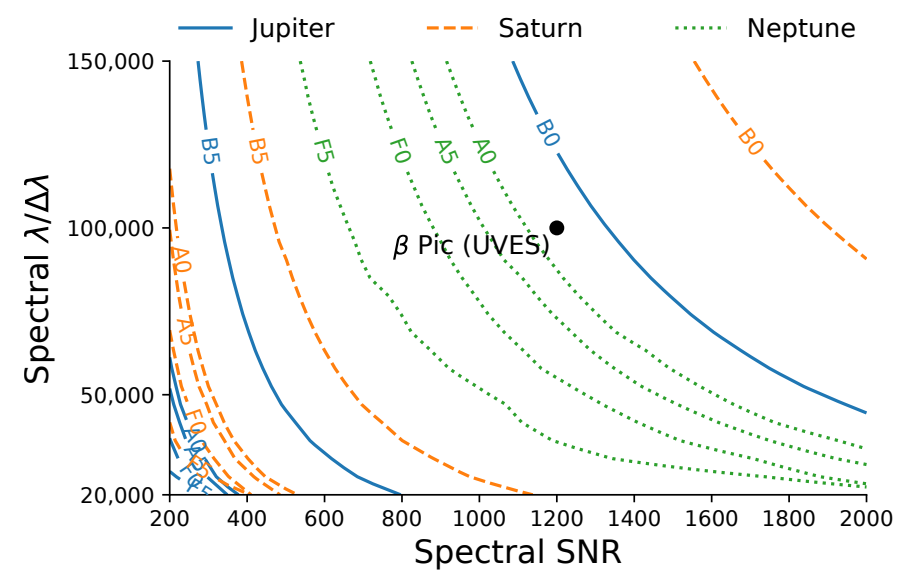

Fig. 8. Contours for different BAF spectral type stars for which a Jupiter-, Saturn-, and Neptune-sized object can be detected at a $S / N>3.0$ following a survey of $20 \mathrm{~min}$ per night for 152 nights. For a higher spectral S/N, smaller objects can be detected. If the spectral resolution is too low, the spectral line can no longer be resolved. For an instrumental setup to the right of a contour, an exoplanet detection is feasible for photon-shot-noise-limited observations. The instrumental setup used for our observations of $\beta$ Pictoris with UVES on the VLT is indicated by the scatter point.

As already seen for $\beta$ Pictoris, stellar activity has a direct impact on our sensitivity. Most late A and F spectral types will suffer the same limitations as they are within the HertzsprungRussel-diagram's instability strip (Gautschy \& Saio 1996). For these objects the stellar pulsation removal procedure described in this work could be applied. For late A and B stars hotter than $\beta$ Pictoris, the prospects are better, as these are outside of the instability strip and also will not have starspots. For stars cooler than $\beta$ Pictoris, starspots could be a problem, as they would show up as similar distortions of the line profile (e.g., for $\alpha$ Cen B, Thompson et al. 2017). However, for these stars (i) we will know whether they are active, (ii) we will see the modulation with the rotation period of the star, and (iii) view the differing impact of the stellar activity between lines, while the planet's signal will be the same for all lines (Dumusque 2018).

Currently, using UVES on the VLT is the only way to get a sufficiently large sample of high $\mathrm{S} / \mathrm{N}$ spectra relatively easily. An advantage of observing bright stars such as $\beta$ Pictoris is that it can be done even during twilight and thus makes optimal use of the telescope. Nonetheless, UVES has not been designed with the aim of surveying the brightest $V<4$ stars in the sky. Firstly, due to the narrow slit width required to get the high spectral resolution $\left(0.3^{\prime \prime}\right)$, in median seeing the slit losses can be a factor of approximately four. Secondly, there are large overhead losses as we integrate for $\sim 15 \mathrm{~s}$, but have to wait $\sim 45 \mathrm{~s}$ before taking the next science image. The relative overheads will increase even further for brighter $(V<4)$ stars, resulting in a further reduced efficiency. Lastly, in this work we combined 16 stellar lines, however, in most cases we can use other techniques, including least-squares deconvolution (LSD; e.g. Donati et al. 1997), to combine a large number of lines and further improve the $\mathrm{S} / \mathrm{N}$. Therefore, we expect that an $\sim 1-2 \mathrm{~m}$ telescope optimized to observe the brightest $(V<4)$ stars in the sky could achieve a similar spectral S/N to our VLT observations of $\beta$ Pic in the same amount of on-sky time. One relatively affordable option would be to refurbish an under-used 1-2m telescope at an observatory where the seeing can be on the order of one or two arcseconds, although an array of newly constructed telescopes is also an option. Each telescope could observe several dozen stars per night and continue the all-sky survey throughout the year. An optimistic back-of-the-envelope calculation of the expected number of detectable transiting companions around a $V<4$ star for such a survey find it is $\sim 0.6$ : the occurrence of transiting $R=R_{\text {Jup }}$ objects is about one in a thousand (Fressin et al. 2013) and for this planet radius we estimate a detection feasible around the $272 V<4 \mathrm{~B}, \mathrm{~A}$, and $\mathrm{F}$ stars. Respectively, the occurrence of transiting $R=R_{\text {Nep }}$ companions is approximately two-in-athousand and we estimate a detection feasible around the 139 $V<4$ A \& F stars. However, we emphasize this estimate does require improvements on the reduction of stellar phenomena such as stellar pulsations and starspots in the future, especially for the A and F stars.

\section{Conclusions}

In this work we demonstrate that the RM effect can be used not only to characterize exoplanetary systems, but also be used for blind spectroscopic transit searches around the brightest rapidly rotating stars in the sky, which are challenging to calibrate with reference star observations. This method is:

- independent of reference stars used for conventional broadband transit surveys and works especially well for strong rotationally broadened stars for which radial velocity measurements are difficult;

- simulated for observations of a typical bright $V \sim 4$ star at $R \sim 100000$, for which we show we are sensitive to Neptunesized objects if the data is photon-shot-noise limited;

- applied to a case study, $\beta$ Pictoris, where the ambiguity between stellar pulsations and exoplanet Doppler shadows constrains our sensitivity to Jupiter-sized objects. However, after our pulsation removal procedure we are sensitive to Saturn-sized objects.

These results are currently the strongest constraints on Jupitersized transiting companions around $\beta$ Pictoris for periods of $15-200$ d with $>50 \%$ coverage.

We have considered it feasible to set up a campaign deploying a set of 1-2 $\mathrm{m}$ telescopes equipped with a high-resolution spectrograph to monitor the brightest stars $(V<4)$ aiming to find the brightest star in the sky with a transiting exoplanet. This is a high-risk endeavor, but with the potential of tremendous scientific reward: the discovery of the brightest star in the sky for detailed exoplanet atmospheric characterization and modeling through transmission spectroscopy.

Acknowledgements. We are thankful to the Leiden exoplanet group members for the fruitful discussions and their supportive criticism that improved the quality of this work. We would also like to thank Remko Stuik for sharing his thoughts on the interpretation of the candidate signals. This research made use of the Python packages Astropy (Astropy Collaboration 2018), SciPy (Jones et al. 2001), NumPy (van der Walt et al. 2011; Oliphant 2015), Matplotlib (Hunter 2007), LMFIT (Newville et al. 2014) and we thank all of their contributors for making their software publicly available. Part of this research was carried out at the Jet Propulsion Laboratory, California Institute of Technology, under a contract with the National Aeronautics and Space Administration.

\section{References}

Alonso, R., Brown, T. M., Torres, G., et al. 2004, ApJ, 613, L153 Anderson, D. R., Temple, L. Y., Nielsen, L. D., et al. 2018, MNRAS, submitted [arXiv: 1809.04897]

Astropy Collaboration (Price-Whelan, A. M., et al.) 2018, AJ, 156, 123

Bakos, G. Á., Noyes, R. W., Kovács, G., et al. 2007, ApJ, 656, 552

Borucki, W. J., Koch, D., Basri, G., et al. 2010, Science, 327, 977

Butters, O. W., West, R. G., Anderson, D. R., et al. 2010, A\&A, 520, L10 
Chen, J., \& Kipping, D. 2017, ApJ, 834, 17

Claret, A. 2000, A\&A, 363, 1081

Collier Cameron, A., Guenther, E., Smalley, B., et al. 2010, MNRAS, 407, 507 de Mooij, E. J. W., Watson, C. A., \& Kenworthy, M. A. 2017, MNRAS, 472 2713

Dekker, H., D’Odorico, S., Kaufer, A., Delabre, B., \& Kotzlowski, H. 2000, in Optical and IR Telescope Instrumentation and Detectors, eds. M. Iye, \& A. F. Moorwood, Proc. SPIE, 4008, 534

Donati, J.-F., Semel, M., Carter, B. D., Rees, D. E., \& Collier Cameron A. 1997, MNRAS, 291, 658

Dumusque, X. 2018, A\&A, 620, A47

Fressin, F., Torres, G., Charbonneau, D., et al. 2013, ApJ, 766, 81

Freudling, W., Romaniello, M., Bramich, D. M., et al. 2013, A\&A, 559, A96

Gautschy, A., \& Saio, H. 1996, ARA\&A, 34, 551

Gray, D. F. 2005, The Observation and Analysis of Stellar Photospheres (Cambridge: Cambridge University Press)

Hunter, J. D. 2007, Comput Sci. Eng., 9, 90

Johnson, M. C., Cochran, W. D., Collier Cameron, A., \& Bayliss, D. 2015, ApJ, 810, L23

Jones, E., Oliphant, T., Peterson, P., et al. 2001-, SciPy: Open source scientific tools for Python

Kenworthy, M. 2017, Nat. Astron., 1, 0099

Koen, C., Balona, L. A., Khadaroo, K., et al. 2003, MNRAS, 344, 1250

Lagrange, A. M., Gratadour, D., Chauvin, G., et al. 2009, A\&A, 493, L21

Lagrange, A. M., Meunier, N., Chauvin, G., et al. 2013, A\&A, 559, A83

Lagrange, A. M., Keppler, M., Meunier, N., et al. 2018, A\&A, 612, A108

Lagrange, A. M., Boccaletti, A., Langlois, M., et al. 2019, A\&A, 621, L8

Lund, M. B., Rodriguez, J. E., Zhou, G., et al. 2017, AJ, 154, 194

Mamajek, E. E., \& Bell, C. P. M. 2014, MNRAS, 445, 2169

McCullough, P. R., Stys, J. E., Valenti, J. A., et al. 2005, PASP, 117, 783
Mol Lous, M., Weenk, E., Kenworthy, M. A., Zwintz, K., \& Kuschnig, R. 2018, A\&A, 615, A145

Newville, M., Stensitzki, T., Allen, D. B., \& Ingargiola, A. 2014, LMFIT: Non-Linear Least-Square Minimization and Curve-Fitting for Python

Oliphant, T. E. 2015, Guide to NumPy (USA: CreateS-pace Independent Publishing Platform)

Pecaut, M. J., \& Mamajek, E. E. 2013, ApJS, 208, 9

Perryman, M. A. C., Lindegren, L., Kovalevsky, J., et al. 1997, A\&A, 323, L49

Pollacco, D. L., Skillen, I., Collier Cameron, A., et al. 2006, PASP, 118, 1407

Rauer, H., Catala, C., Aerts, C., et al. 2014, Exp. Astron., 38, 249

Ricker, G. R., Winn, J. N., Vanderspek, R., et al. 2014, in Space Telescopes and Instrumentation 2014: Optical, Infrared, and Millimeter Wave, Proc. SPIE, 9143914320

Seager, S., \& Deming, D. 2010, ARA\&A, 48, 631

Talens, G. J. J., Albrecht, S., Spronck, J. F. P., et al. 2017a, A\&A, 606, A73

Talens, G. J. J., Spronck, J. F. P., Lesage, A. L., et al. 2017b, A\&A, 601, A11

Talens, G. J. J., Justesen, A. B., Albrecht, S., et al. 2018, A\&A, 612, A57

Temple, L. Y., Hellier, C., Albrow, M. D., et al. 2017, MNRAS, 471, 2743

Thompson, A. P. G., Watson, C. A., de Mooij, E. J. W., \& Jess, D. B. 2017, MNRAS, 468, L16

Triaud, A. H. M. J. 2017, The Rossiter-McLaughlin Effect in Exoplanet Research (London: Springer), 2

van der Walt, S., Colbert, S. C., \& Varoquaux, G. 2011, Comput. Sci. Eng., 13, 22

van Leeuwen F., ed. 2007, Hipparcos, the New Reduction of the Raw Data (Berlin: Springer), Astrophysics and Space Science Library, 350

Wang, J. J., Graham, J. R., Pueyo, L., et al. 2016, AJ, 152, 97

Wenger, M., Ochsenbein, F., Egret, D., et al. 2000, A\&AS, 143, 9

Wheatley, P. J., West, R. G., Goad, M. R., et al. 2018, MNRAS, 475, 4476

Zwintz, K. 2018, 3rd BRITE Science Conference, Vol. 8, 161 